\title{
Severe dystonia, cerebellar atrophy, and cardiomyopathy likely caused by a missense mutation in TOR1AIP1
}

Imen Dorboz ${ }^{1 \dagger}$, Marie Coutelier ${ }^{2,3,4,5,6 \dagger}$, Anne T Bertrand ${ }^{7,8}$, Jean-Hubert Caberg ${ }^{9}$, Monique Elmaleh-Bergès ${ }^{10}$, Jeanne Lainé $e^{7,8,11}$, Giovanni Stevanin ${ }^{2,3,4,5}$, Gisèle Bonne ${ }^{7,8,12}$, Odile Boespflug-Tanguy ${ }^{1,13}$ and Laurent Servais ${ }^{13,14,15^{*}}$

\begin{abstract}
Background: Dystonia, cerebellar atrophy, and cardiomyopathy constitute a rare association.

Methods: We used homozygosity mapping and whole exome sequencing to determine the mutation, western blot and immunolabelling on cultured fibroblasts to demonstrate the lower expression and the mislocalization of the protein.

Results: We report on a boy born from consanguineous healthy parents, who presented at three years of age with rapidly progressing dystonia, progressive cerebellar atrophy, and dilated cardiomyopathy. We identified regions of homozygosity and performed whole exome sequencing that revealed a homozygous missense mutation in TOR1AIP1. The mutation, absent in controls, results in a change of a highly conserved glutamic acid to alanine. TOR1AIP1 encodes lamina-associated polypeptide 1 (LAP1), a transmembrane protein ubiquitously expressed in the inner nuclear membrane. LAP1 interacts with torsinA, the protein mutated in DYT1-dystonia. In vitro studies in fibroblasts of the patient revealed reduced expression of LAP1 and its mislocalization and aggregation in the endoplasmic reticulum as underlying pathogenic mechanisms.

Conclusions and relevance: The pathogenic role of TORIAIP1 mutation is supported by a) the involvement of a highly conserved amino acid, b) the absence of the mutation in controls, c) the functional interaction of LAP1 with torsinA, and d) mislocalization of LAP1 in patient cells. Of note, cardiomyopathy has been reported in LAP1-null mice and in patients with the TORIAIP1 nonsense mutation. Other cases will help delineate the clinical spectrum of LAP1-related mutations.
\end{abstract}

Keywords: Dystonia, Cerebellar atrophy, Torsin, Nuclear membrane

\section{Background}

Dystonia, cerebellar atrophy and cardiomyopathy constitute a very rare association, observed in rare mitochondrial disease and organic acidemia. We report on a boy with early onset dystonia, for whom work-up performed in several institutions failed to reach a diagnosis.

\footnotetext{
* Correspondence: Iservais@institut-myologie.org

${ }^{\dagger}$ Equal contributors

${ }^{13}$ Service de neurologie pédiatrique et des maladies métaboliques, Hôpital Robert Debré, Assistance Publique des Hôpitaux de Paris, 75019 Paris, France

${ }^{14}$ Centre de Référence des Maladies Neuromusculaires, Hôpital de La

Citadelle, 4000 Liège, Belgium

Full list of author information is available at the end of the article
}

\section{Methods}

We performed homozygosity mapping in the patient by using the Human Omni2.5 array (Illumina), and wholeexome sequencing (IntegraGen), using the SureSelect V4 capture kit (Agilent) and the HighSeq2000 sequencer (Illumina) [1].

For cell cultures work up, skin fibroblasts were maintained in DMEM (Life Technologies) with 10\% FBS and penicillin/streptomycin. Cells were either plated on glass coverslips for immunofluorescence or pelleted for protein analysis. Cells were imaged using an Olympus FV1000 confocal microscope, and electron microscopy was performed as described $[2,3]$.

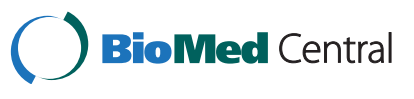

(C) 2014 Dorboz et al.; licensee BioMed Central. This is an Open Access article distributed under the terms of the Creative Commons Attribution License (http://creativecommons.org/licenses/by/4.0), which permits unrestricted use, distribution, and reproduction in any medium, provided the original work is properly credited. The Creative Commons Public Domain Dedication waiver (http://creativecommons.org/publicdomain/zero/1.0/) applies to the data made available in this article, unless otherwise stated. 
For Protein extraction and immunoblotting, Cell pellets were lysed in Protein Lysis Buffer. Proteins $(20 \mu \mathrm{g})$ were separated by SDS-PAGE and transferred to nitrocellulose. Membranes were blocked with blocking buffer and incubated with primary or secondary antibodies diluted in the same buffer [3] (secondary Alexa-conjugates antibodies: Life Technologies; HRP-conjugated antibodies: Jackson ImmunoResearch).

\section{Ethic statement}

Written informed consent was obtained from the patient's legal guardian(s) for gene analysis and publication of this case report and any accompanying images. A copy of the written consent is available for review by the Editor-in-Chief of this journal.

\section{Result and discussion}

The boy was born after an uneventful pregnancy to healthy Moroccan parents who are second-degree related (Figure 1A). Familial history was irrelevant. Motor milestones were normal until the age of three, when the boy began to fall frequently and to develop progressive dystonia that first affected lower limbs and spread rapidly to upper limbs, trunk, and face. He lost ambulation

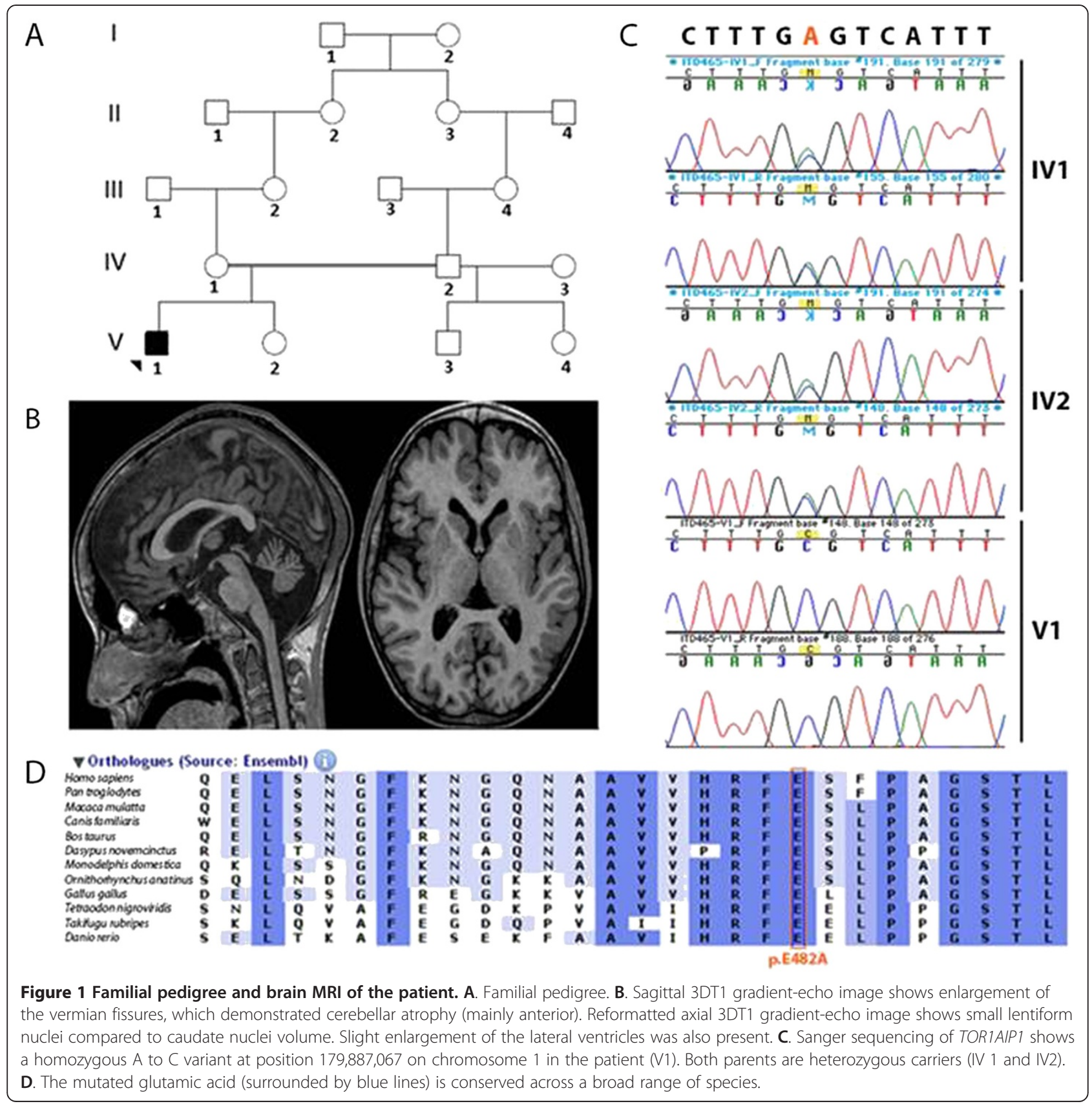


at the age of 5 . Dystonia was permanent, exacerbated by illness or stress, and by the age of 10 was completely generalized and extremely painful. The child spent most of the day and night crying and was unable to maintain a sufficient level of food and liquid intake (Additional file 1: Video S1). Severe contractures of the Achilles tendons and permanent feet deformations were noticed. Cerebellar maneuvers on upper limbs were normal. There was no ophthalmoplegia, ptosis, or pyramidal signs. Intelligence appeared normal. Medications, including paracetamol, opioids, benzodiazepines (diazepam, clonazepam, clobazam), L-Dopa, phenobarbitone, levetiracetam, piracetam, vigabatrin, propanolol, trihexyphenidyl hydrochloride, dantrolene, baclofen, carbamazepine, valproic acid, topiramate, chlorpromazine, amytriptiline, tetrabenazine, 1,3,5-benzenetriol, and magnesium sulfate, were tried unsuccessfully. The child's pain was temporally relieved by intravenous chlorpromazine. Nabiximols led to a dramatic relief of pain and dystonia, and allowed the patient to re-acquire the ability to swallow, sit, and perform some voluntary movement (Additional file 2: Video S2). Attempts to wean the patient off medication led to rapid recurrence of pain. At the age of 14, a dilated cardiomyopathy was diagnosed, and ejection fraction rapidly decreased leading to death at the age of 17 .

Prior to death, brain MRIs showed progressive global cerebellar atrophy (Figure 1B). Monovoxel MR spectroscopy of the left basal ganglia revealed a reduced NAA/ $\mathrm{Cr}$ ratio indicative of neuronal loss without iron accumulation. Brain PET scans, electroencephalographic recordings, somatosensory evoked potentials, audition and fundus examination, electroneurography, liver and kidney echographies were unremarkable. Muscle biopsy, performed at the age of 6 , revealed no abnormalities or biochemical deficits. Glucose, proteins, lactate, blood cell count, and neurotransmitters levels in the CSF were normal. Analyses for each of the following, performed at least once, were normal: blood cell count, ASAT, ALAT, CK, urea, creatinine, cholesterol, triglyceride, arterial lactate and pyruvate levels, ceruloplasmin, cupremia and cupruria, alpha fetoprotein, very long chain fatty acids and long chain fatty acids, biopterin, urine creatine and guanidinoacetate, amino acid (blood and urine) and organo acid (urine) chromatography, high-resolution caryotype, glucocerebrosidase, galactocerebrosidase, $\beta$-galactosidase, $\alpha$-N-acetylgalacosaminidase, aryl sulfatase A, hexosaminidase $\mathrm{A}$ and $\mathrm{B}, \alpha$-glucosaminidase, $\beta$-glucuronidase, $\alpha$-mannosidase, $\beta$-mannosidase, $\alpha$-neuraminidase, acid sphingomyelinidase mucopolysaccharidoses and oligosaccharidoses, and sialotransferrin. No acanthocytosis was present on any of several blood smears.

No mutations were identified in DYT1, PANK2, PLA2G6, SCA17, or APTX, genes known to be involved in neurodegenerative diseases with cerebellar atrophy and/or dystonia. Given consanguinity in the family, we assumed a recessive mode of inheritance and predicted the causative variant would fall in a region of homozygosity.

Homozygosity mapping revealed six homozygous regions greater than $2 \mathrm{Mb}$. Whole exome sequencing produced 142 million reads, $98 \%$ of which could be aligned to the targeted sequence. Mean coverage of the targeted sequence was 77 fold. 56687 SNVs and 7265 indels were called. Only nine homozygous variants with frequency below $1 \%$ in internal (IntegraGen) and public databases (dbSNP 132; HapMap; 1,000 Genomes Project) were filtered out of these. Sequencing in the patient and in family members (primers available upon request) reduced the number of candidates to four genes, present at the homozygous state in the patient, and at the heterozygous state in the parents: ALMS1, B3GALT1, ZNF804B, and TOR1AIP1.

The $\mathrm{A}>\mathrm{C}$ missense variant $(\mathrm{c} .1448 \mathrm{~A}>\mathrm{C})$ at position 179,887,067 on chromosome 1q25.1-1q25.3 in TOR1AIP1 (NM_015602), located in a 6.8-Mb homozygosity region, resulted in replacement of a highly conserved glutamic acid with alanine at amino acid 482 (GERP++ score 5.96; PhyloP score 2.285) (Figure 1C,D). Furthermore, pathogenicity predictions were deleterious in Align GVGD, Polyphen-2, SIFT, and MutationTaster analyses. On the contrary, the variants in ALMS1 (NM_015120; c.2202T>A/p.S732R), B3GALT1 (c.192A>T/p.E64D, NM_ 020981) and ZNF804B (c3118C>A/p.L1040I, NM_181646), were predicted to be benign by at least three of the abovementioned programs. GERP++ and PhyloP scores were lower for the ZNF804B variant (GERP++ score 4.15, PhyloP score 1.467), and even negative for the ALMS1 and B3GALT1 variants. There was thus a strong bioinformatic convergence towards the pathogenic character of the TOR1AIP1 variant only. In addition, the phenotype of this patient was divergent from that of Alström syndrome (OMIM \#203800) patients who have mutations in ALMS1. TOR1AIP1 encodes LAP1, a type II transmembrane protein. LAP1 interacts with torsinA (encoded by TOR1A gene), which is mutated in autosomal dominant dystonia (DYT1; OMIM \#12810) [4]. The amino acid mutated in our patient is located in the luminal domain, which interacts with torsinA. This domain is common to the three isoforms and has significant homology with LULL1, another protein that interacts with torsinA. This variant was not observed in any of 100 ethnically matched controls and was absent from $>6500$ exomes at the Exome Variant Server.

To gain insight into the pathogenicity of the TOR1AIP1 mutation, we evaluated primary skin fibroblasts from the patient. By western blot, a strong reduction in the expression of LAP1 isoforms was observed relative to control cells (Figure 2A). Immunolabeling revealed a significant reduction in LAP1 staining in the nuclear 


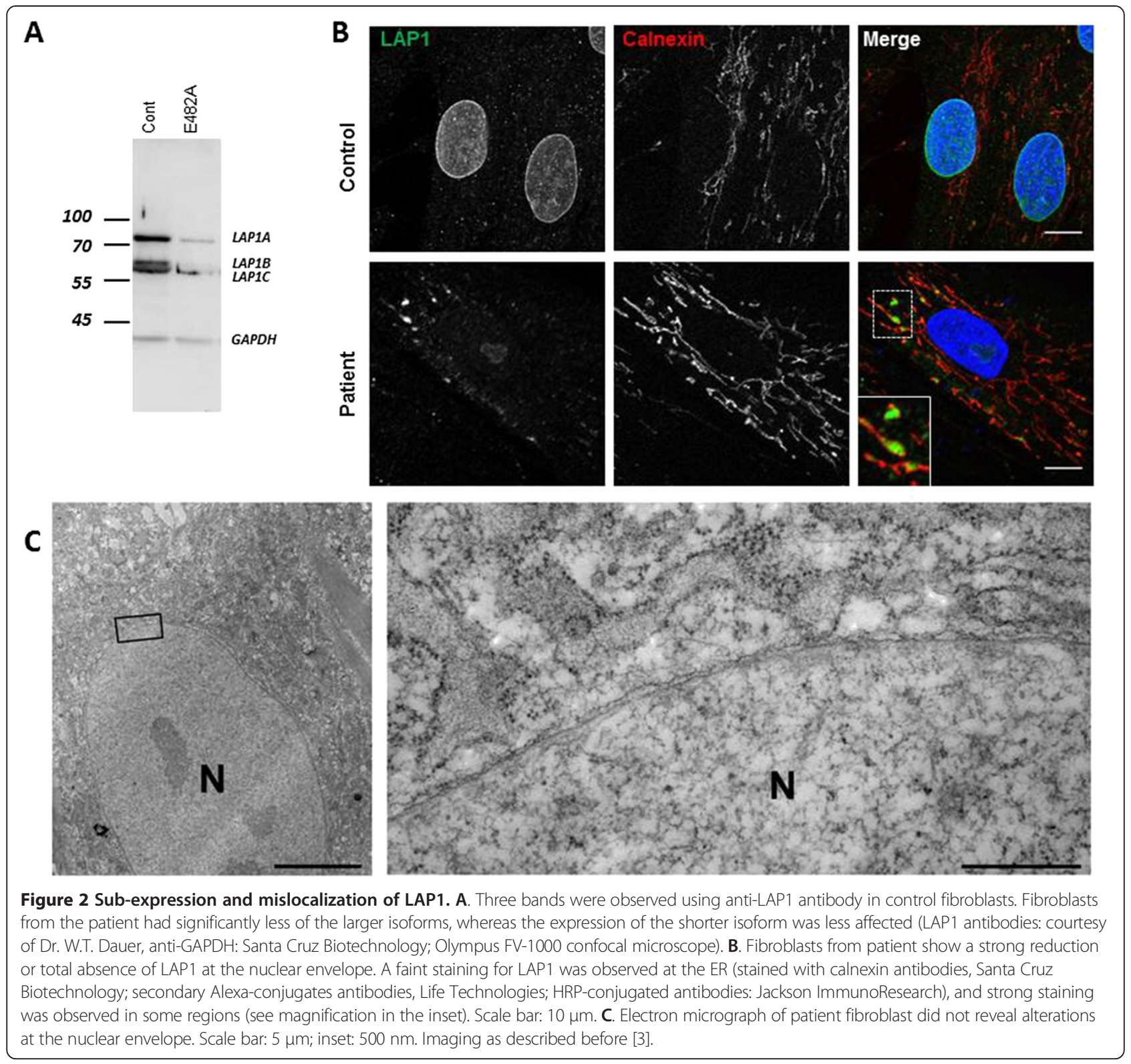

envelope of patient cells (Figure 2B). Although the endoplasmic reticulum was generally faintly stained, some areas showed accumulation of LAP1 (Figure 2B), indicating mislocalization of the mutated LAP1. No defects in A-type or B-type lamins, SUN1, SUN2, or nesprin-1 or 2 protein localization were observed (data not shown). Similarly, no blebs [4] in nuclear envelopes were observed by electron microscopy in patient cells (Figure 2C). TorsinA is not normally expressed in fibroblasts, so we were not able to determine if this protein was mislocalized.

We have searched TOR1AIP1 mutation in 10 additional patients with dystonia during childhood: Five with primary dystonia associated with cerebellar atrophy and 5 with primary cerebellar ataxia with progressive cerebellar atrophy and severe dystonia. We did not find mutations, which seems to indicate that TOR1AIP1 mutation is not a common cause of dystonia of unknown origin.

To our knowledge, cerebellar atrophy, dystonia, and dilated cardiomyopathy are very rarely associated, except in some mitochondrial disease or organic acidemia. Our patient did not suffer from organic acidemia and mitochondrial disorders were unlikely given normal blood and CSF lactate levels, NMR spectroscopy, and muscular biopsy. Cerebellar atrophy and dystonia are observed in neurodegenerative diseases, such as Wilson disease, acanthocytosis, gangliosidosis [5], and Niemann Pick type $\mathrm{C}[6]$ and in some cases of spinocerebellar ataxias, particularly SCA17 [7]. These were ruled out in the patient. TUBB4A mutations have been recently involved in patients with dystonia, cerebellar and basal ganglia 
atrophy and hypomyelinating leukodystrophies (HABC syndrome) [8]. In addition to the absence of white matter abnormalities in our case, exome analysis ruled out TUBB4A mutations. In the clinical work up, other causes of dystonia (such as DYT1 and PANK2 mutation) or cerebellar atrophy (such as PLA2G6 mutation) were also ruled out. Much older patients who have presented with the association of a less severe dystonia and a cerebellar atrophy have been described [9]. Extreme painfull dystonia was one of the primary clinical features observed when we first evaluated the child at the age of 11 . Absence of effect of any usual oral medications tried by us and others, and the dramatic improvement upon administration of nabiximols, a cannabinoid derived from cannabis plant [10], remains very enigmatic but as been reported in adults with central pain and paroxysmal dystonia [11].

LAP1, encoded by TOR1AIP1, induces the ATPase activity of torsinA [12], the protein mutated in DYT1, an autosomal dominant dystonia with purely neurological phenotype. LAP1 is the only protein with such function at the nuclear envelope described so far, and hence its mislocalization likely leads to a dramatic loss of function of torsin A and probably of other torsin family proteins [12]. Mice lacking LAP1 have abnormal nuclear membranes that form blebs visible by electron microscopy in all cell types [4]. In mice lacking torsinA, blebs are only present in neurons, probably because of the high level of torsinB in non-neuronal cells [13]. Such blebs were not observed in patient's fibroblasts. The mutated LAP1 expressed by the patient may retain some functions required for the structure of the nuclear envelope. Our case appears much more severe than DYT1, not only because of the severity of dystonia itself, but because of the cardiac involvement, similar to the phenotype of $\operatorname{Tor} 1 a^{\Delta E / \Delta E}$ mice [4] and with disorders of nuclear membrane such as laminopathies [3]. Recently, cardiomyopathy has been demonstrated in a tissue-specific mutant lacking LAP1 [14].

One Turkish family with a homozygous nonsense mutation was recently reported [15]. Interestingly, affected patients also present with a cardiomyopathy, although less severe than that observed in our patient, and associated with a limb-girdle muscular dystrophy.

\section{Conclusions}

The present observation is unique, and, therefore, it cannot be completely ruled out that the phenotype is not related to a mutation in a region not covered by exome sequencing. However, we believe that the association between the previously unreported phenotype of the patient and the mutation is very likely. First, clinical evaluations failed to identify another likely cause. Second, the mutation affects a highly conserved amino acid, is predicted to be pathogenic, is absent in 100 ethnically matched controls and in $>6500$ exomes, and affects a protein that interacts with a protein involved in dystonia. Third, the patient phenotype correlates with that of mice lacking LAP1 [14], and functional explorations of patient cells revealed reduced LAP1 expression and mislocalization. It is known that adequate localization of LAP1 is crucial for its function [12]. Finally, another family has been reported with nonsense mutation leading to cardiomyopathy [15]. For these different reasons, we believe that sequencing of TOR1AIP1 should be performed in patients with similar peculiar phenotypic associations.

\section{Availability of supporting data}

All supporting data are available within the limits of patient's confidentiality.

\section{Additional files}

Additional file 1: Video S1. Severe and painful dystonia before starting nabiximols.

Additional file 2: Video S2. Video taken by the parents after three months of nabiximols. The father asks the child to move his leg.

\section{Competing interests}

The authors declare that they have no competing interests.

\section{Authors' contributions}

ID and MC conducted gene analysis, drafted the paper, and approved the final version. ATB designed and conducted the in vitro experiments and approved the final version. $\mathrm{JHC}$ conducted gene analysis and approved the final version. MEB performed the MRI and participated in patient follow-up. She approved the final version. JL designed and conducted the in vitro experiments and approved the final version. GS supervised gene analysis and approved the final version. GB designed the in vitro experiments and approved the final version. OBT supervised gene analysis and approved the final version. LS followed the patient, designed in vitro experiments, drafted the paper and approved the final version.

\section{Acknowledgments}

The authors thank the patient and his family for their strong support to report this case, Alexandra Durr for helpful comments, William T. Dauer for the anti-LAP1 antibody, the DNA and Cell Bank of the Institut du Cerveau et de la Moelle épinière, and the Fond National de la Recherche Scientifique for the fellowship attributed to M. C. (aspirant FNRS). This work was financially supported by the French National Agency for Research (ANR), the Institut national de la santé et de la recherche médicale (INSERM), the Université Paris Diderot-Sorbonne Paris Cité, DHU PROTECT and the ELA Fondation (post doc fellowship for I.D.), the Université Pierre et Marie Curie Paris 06, the Centre National de la Recherche Scientifique (CNRS), the Association Française contre les Myopathies (AFM), the Verum Foundation, the Fondation Roger de Spoelberch, and the program "Investissements d'avenir" ANR-10-IAlHU-06.

\section{Author details}

${ }^{1}$ Inserm U1141, Université Paris Diderot-Sorbonne Paris Cité, DHU PROTECT, Paris F-75019, France. ${ }^{2}$ Inserm, U1127, Paris F-75013, France. ${ }^{3}$ CNRS, UMR 7225, Paris 75013, France. ${ }^{4}$ Université Pierre et Marie Curie - Paris 6, UMR_S 1127, Institut du Cerveau et de la Moelle épinière, CHU Pitié-Salpêtrière, 75013 Paris, France. ${ }^{5}$ Laboratoire de Neurogénétique, Ecole Pratique des Hautes Etudes, Institut du Cerveau et de la Moelle épinière, CHU Pitié-Salpêtrière, 75013 Paris, France. ' Laboratoire de Génétique Humaine, Institut de Duve, UCL, 1200 Bruxelles, Belgium. 'Inserm, U974, Paris F-75013, France. ${ }^{8}$ Université Pierre et Marie Curie - Paris 6, UM 76; CNRS, UMR 7215; Institut de Myologie, Paris F-75013, France. ${ }^{9}$ Service de génétique, CHU du 
Sart Tilman, Liège, Belgium. ${ }^{10}$ Service d'Imagerie Pédiatrique, Hôpital Robert Debré, 75019 Paris, France. ${ }^{11}$ Département de Physiologie, Université Pierre et Marie Curie - Paris 6, Site Pitié-Salpêtrière, Paris F-75013, France. ${ }^{12}$ AP-HP, Groupe Hospitalier Pitié-Salpêtrière, U.F. Cardiogénétique et Myogénétique, Service de Biochimie Métabolique, Paris F-75013, France. ${ }^{13}$ Service de neurologie pédiatrique et des maladies métaboliques, Hôpital Robert Debré, Assistance Publique des Hôpitaux de Paris, 75019 Paris, France. ${ }^{14}$ Centre de Référence des Maladies Neuromusculaires, Hôpital de La Citadelle, 4000 Liège, Belgium. ${ }^{15}$ Institut de Myologie, Bâtiment Babinski, Hôpital de La Pitié Salpêtrière, 48/83 boulevard de l'Hôpital, 75013 Paris, France.

Received: 21 July 2014 Accepted: 28 October 2014

Published online: 26 November 2014

\section{References}

1. Boukhris A, Schule R, Loureiro JL, Lourenço CM, Mundwiller E, Gonzalez MA, Charles P, Gauthier J, Rekik I, Acosta Lebrigio RF, Gaussen M, Speziani F, Ferbert A, Feki I, Caballero-Oteyza A, Dionne-Laporte A, Amri M, Noreau A, Forlani S, Cruz VT, Mochel F, Coutinho P, Dion P, Mhiri C, Schols L, Pouget J, Darios F, Rouleau GA, Marques W Jr, Brice A, et al: Alteration of ganglioside biosynthesis responsible for complex hereditary spastic paraplegia. Am J Hum Genet 2013, 93(1):118-123.

2. Bertrand AT, Chikhaoui K, Ben Yaou R, Bonne G: Clinical and genetic heterogeneity in laminopathies. Bioch Soc Trans 2011, 39(6):1687-1692.

3. Cattin ME, Bertrand AT, Schlossarek S, Le Bihan MC, Skov Jensen S, Neuber C, Crocini C, Maron S, Lainé J, Mougenot N, Varnous S, Fromes Y, Hansen A, Eschenhagen T, Decostre V, Carrier L, Bonne G: Heterozygous LmnadelK32 mice develop dilated cardiomyopathy through a combined pathomechanism of haploinsufficiency and peptide toxicity. Hum Mol Genet 2013, 22(15):3152-3164.

4. Kim CE1, Perez A, Perkins G, Ellisman MH, Dauer WT: A molecular mechanism underlying the neural-specific defect in torsinA mutant mice. Proc Natl Acad Sci U S A 2010, 107(21):9861-9866.

5. De Carvalho Aguiar PM, Ozelius L: Classification and genetics of dystonia. Lancet Neurol 2002, 1(5):316-325.

6. Vanier MT, Millat G: Niemann-pick disease type C. Clin Genet 2003, 64(4):269-281

7. Stevanin G, Brice A: Spinocerebellar ataxia 17 (SCA17) and Huntington's disease-like 4 (HDL4). Cerebellum 2008, 7(2):170-178.

8. Hamilton EM, Polder E, Vanderver A, Naidu S, Schiffmann R, Fisher K, Raguž AB, Blumkin L, H-ABC Research Group, van Berkel CG, Waisfisz Q, Simons C, Taft RJ, Abbink TE, Wolf NI, van der Knaap MS: Hypomyelination with atrophy of the basal ganglia and cerebellum: further delineation of the phenotype and genotype-phenotype correlation. Brain 2014, 137(7):1921-1930.

9. Le Ber I, Clot F, Vercueil L, Camuzat A, Viémont M, Benamar N, De Liège $P$, Ouvrard-Hernandez AM, Pollak P, Stevanin G, Brice A, Dürr A: Predominant dystonia with marked cerebellar atrophy: a rare phenotype in familial dystonia. Neurology 2006, 67(10):1769-1773.

10. Borgelt LM, Franson KL, Nussbaum AM, Wang GS: The pharmacologic and clinical effects of medical cannabis. Pharmacotherapy 2013, 33(2):195-209.

11. Koppel BS, Brust JC, Fife T, Bronstein J, Youssof S, Gronseth G, Gloss D: Systematic review: efficacy and safety of medical marijuana in selected neurologic disorders: report of the Guideline Development

Subcommittee of the American Academy of Neurology. Neurology 2014, 82(17):1556-1563.

12. Zhao C, Brown RS, Chase AR, Eisele MR, Schlieker C: Regulation of Torsin ATPases by LAP1 and LULL1. Proc Natl Acad Sci U S A 2013, 110(17):1545-1554

13. Jungwirth M, Dear ML, Brown P, Holbrook K, Goodchild R: Relative tissue expression of homologous torsinB correlates with the neuronal specific importance of DYT1 dystonia-associated torsinA. Hum Mol Genet 2010, 19(5):888-900.

14. Shin JY, Méndez-López I, Wang Y, Hays AP, Tanji K, Lefkowitch JH, Schulze PC, Worman HJ, Dauer WT: Lamina-associated polypeptide-1 interacts with the muscular dystrophy protein emerin and is essential for skeletal muscle maintenance. Dev Cell 2013, 26(6):591-603.

15. Kayman-Kurekci G, Talim B, Korkusuz P, Sayar N, Sarioglu T, Oncel I, Sharafi P, Gundesli H, Balci-Hayta B, Purali N, Serdaroglu-Oflazer P, Topaloglu H, Dincer P: Mutation in TOR1AIP1 encoding LAP1B in a form of muscular dystrophy: a novel gene related to nuclear envelopathies. Neuromuscul Disord 2014, 24(7):624-633.

\section{Submit your next manuscript to BioMed Central and take full advantage of:}

- Convenient online submission

- Thorough peer review

- No space constraints or color figure charges

- Immediate publication on acceptance

- Inclusion in PubMed, CAS, Scopus and Google Scholar

- Research which is freely available for redistribution

Submit your manuscript at www.biomedcentral.com/submit
C) Biomed Central 